\title{
Complementary Analytical TEM Analysis of Perpendicular Recording Media
}

\author{
Soyoung Park, ${ }^{1,3}$ N.T. Nuhfer, ${ }^{1}$ D.E. Laughlin, ${ }^{1,2,3}$ and J.-G.Zhu ${ }^{1,2,3}$ \\ * Materials Science and Engineering, Carnegie Mellon University, Pittsburgh, PA 15213 \\ ** Electrical and Computer Engineering, Carnegie Mellon University, Pittsburgh, PA 15213 \\ *** Data Storage System Center, Carnegie Mellon University, Pittsburgh, PA 15213
}

Extending the areal recording density of CoCrPt:oxide perpendicular recording media significantly depends on microstructural engineering. It is crucial for media to have small magnetic grains which are isolated by a non magnetic oxide. Currently, the signal to noise ratio (SNR) does not seem to improve when the grain size is below $6 \mathrm{~nm}$. It has been speculated that the "cavity-like" features found in the center of magnetic grains in the recording layer can be mainly responsible for the stagnation in SNR for the small grain media [1-2]. To solve this problem, it is necessary to identify the nature of such cavities in the grains and understand why such phenomenon occurs. We analyzed a commercial $\mathrm{CoCrPt}: \mathrm{SiO}_{2}$ perpendicular media using three analytical techniques which supplement bright field in conventional transmission electron microscopy (BF CTEM), namely (1) high angle annular dark field scanning transmission electron microscopy (HAADF STEM) imaging, (2) energy dispersive x-ray spectroscopy (EDXS) mapping (in STEM mode), and (3) elemental mapping and thickness mapping using electron energy loss spectroscopy (EELS) in energy filtered TEM (EFTEM).

To avoid misinterpretation of grain topology or artifacts of imaging, it is important to understand the complexity of materials system and the physics of each technique. First, the perpendicular media consists of multilayer, which hinders the ability to display the information only for the recording layer in a plan view image (Fig 1(a)). Especially, the observed media has a capping layer on top of the recording layer. However, the ion milling procedure seems to help to mill off the other layers selectively as illustrated in Fig 1(b). Cross section images in Fig 1(c) also incorporate many grains which makes difficult to define the topology of a grain as shown in Fig 1(d). Fig 2 shows that HAADF reveals the presence of cavities clearly while bright field image does not. Secondly, EELS analysis of each element requires caution to acquire reliable mapping. Since the media consists of multiple elements in range of composition, it is crucial to consider various operation conditions: accurate extraction of an appropriate energy loss peak by a proper background subtraction method, selection of the correct collection angle to avoid the dominant contribution of diffracted electrons as well as specimen thickness, and so on. This is because not only the energy loss peaks can be overlapped each other, but also SNR of Cr, $\mathrm{Pt}$, and $\mathrm{O}$ edges, and Si can be not high enough to obtain the reliable mappings due to their own different reasons. Thirdly, thickness mapping $(\mathrm{t} / \lambda$ where $\mathrm{t}$ is thickness and $\lambda$ is inelastic scattering mean free path) for grain topology also can be affected by the chemical distribution information due to the large difference of inelastic scattering mean free path among elements. Lastly, HAADF images appear to include thickness contributions as well as elemental contributions. A series of images taken at different collection angles showed that the same feature at any angle.

The careful complementary analysis confirmed that a cavity is Co,Pt-deficient, and $\mathrm{Si}, \mathrm{Cr}, \mathrm{O}-$ rich region, where it can be interpreted as an onset of a subgrain boundary similar to other oxide grain boundary. It suggests that one-to-one grain growth with Ru dome is no longer preferred at high oxide content media.[3] When sputtered, some of oxide also goes to the center 
of grains, which leads to 3-4 different grains are nucleated in one Ru dome. We are working on ways to prevent this from happening and hence improve the SNR of small grain media.

\section{References}

[1] J.-G. Zhu et el, IEEE Trans. on Magn. 45, (2009) 2 (in Press)

[2] D.E. Laughlin et el, J. of Appl. Phys. 105, (2009) 1 (in Press)

[3] J. D. Risner et el, Microsc. Microanal. 13, (2007) 70

[4] This work was supported by Williams Advanced Materials, and the Data Storage System Center of Carnegie Mellon University.

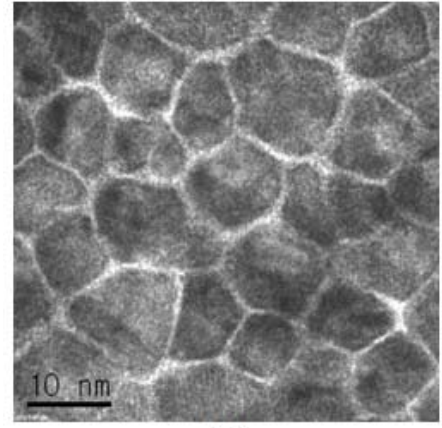

(a)

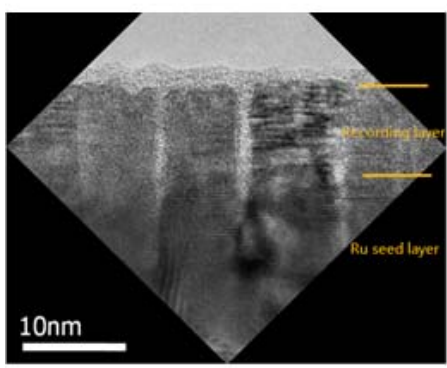

(c)

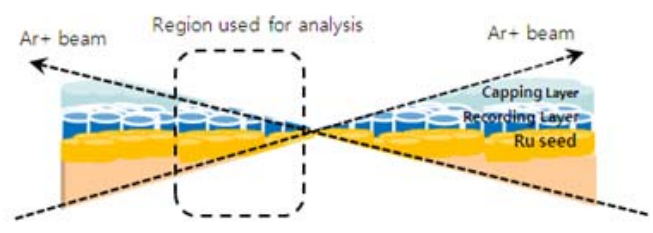

(b)

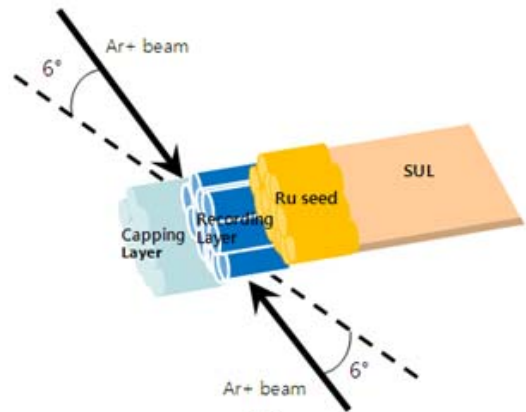

(d)

FIG. 1. (a) BF plan view image showing recording layer consisting of magnetic grains isolated by oxide. (b) Schematic Precision Ion Polishing System (PIPS) procedure of plan view illustrating how to obtain the region of analysis. (c) Cross section view showing grains are generally isolated and conform Ru dome structure, however, it is hard to distinguish one-to-one growth because information still is obtained from multi grains. (d) Schematic of PIPS procedure illustrating how cross section images contain multigrain information.

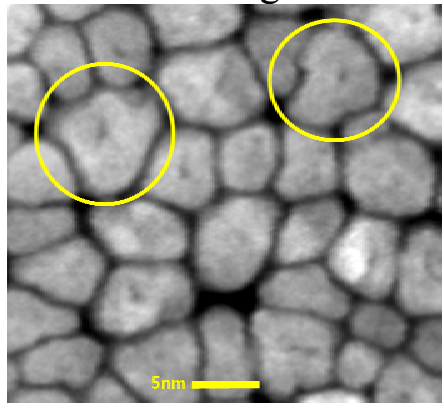

FIG. 2. HAADF image displaying region with cavities that is not clearly seen in BF. 